\title{
Correction to: Antidepressant-Like Action of Single Facial Injection of Botulinum Neurotoxin A is Associated with Augmented 5-HT Levels and BDNF/ERK/CREB Pathways in Mouse Brain
}

\author{
Yang $\mathrm{Li}^{1} \cdot$ Jing Liu ${ }^{1} \cdot \mathrm{Xu} \mathrm{Liu}{ }^{1,2} \cdot$ Cun-Jin Su${ }^{1,2,3} \cdot$ Qi-Lin Zhang ${ }^{1} \cdot$ \\ Zhi-Hong Wang ${ }^{2}$ Lei-Fang Cao ${ }^{4} \cdot$ Xue-Yan Guo $^{1} \cdot$ Ya Huang $^{2} \cdot$ Weifeng Luo ${ }^{1} \cdot$ \\ Tong Liu ${ }^{1,2,5}$
}

Published online: 8 June 2019

(C) Shanghai Institutes for Biological Sciences, CAS 2019

\section{Correction to: Neurosci. Bull.}

https://doi.org/10.1007/s12264-019-00367-8

In the original publication, Figure $4 \mathrm{G}$ was incorrectly published. The correct version of Figure $4 \mathrm{G}$ is presented in this correction. This correction does not affect the conclusions of the paper.

Yang Li, Jing Liu, Xu Liu and Cun-Jin Su have contributed equally to this work.

The original article can be found online at https:// doi.org/10.1007/s12264-019-00367-8.

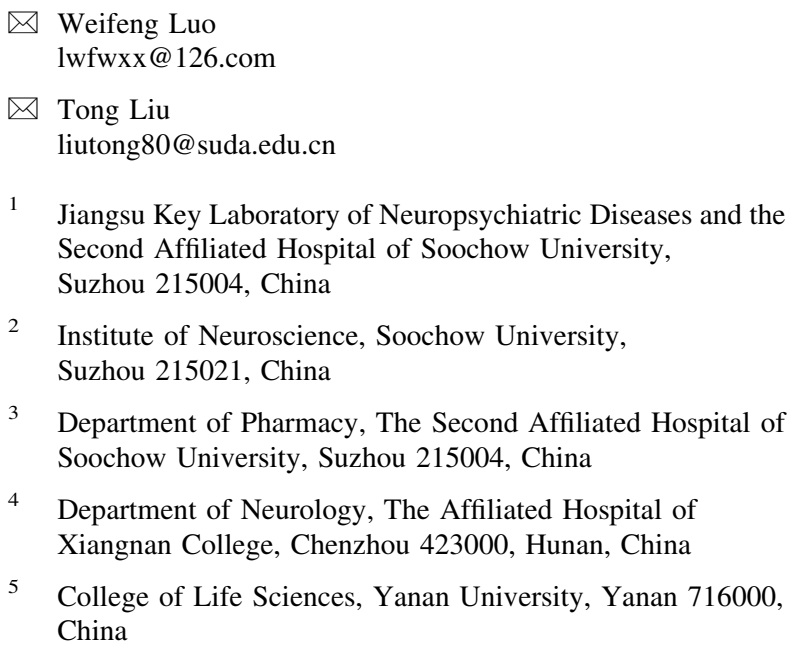

4 Department of Neurology, The Affiliated Hospital of Xiangnan College, Chenzhou 423000, Hunan, China

5 College of Life Sciences, Yanan University, Yanan 716000, China 
Fig. 4 BoNT/A treatment attenuates depression-like behaviors in SRS mice. A The experimental procedure. B Body weight. C, D Immobility time in the FST $(\mathbf{C})$ and TST (D) was increased in SRS-treated mice. $* P<0.05, * * P<0.01$, $* * * P<0.001$ vs. controls. Student's $t$-test; $n=10-22 /$ group. E There was no significant difference in sucrose preference between SRS and control mice. Student's $t$-test;

$n=7-10$ /group. F, G Immobility time in the FST $(\mathbf{F})$ and TST (G) at different time points after BoNT/A injection in SRS mice. $\mathrm{H}$ Body weight at different time points after BoNT/A injection in SRS mice. $* P<0.05$,

$* * P<0.01, * * * P<0.001$ vs. controls (two-way ANOVA with post hoc Bonferroni test). ${ }^{\#} P<0.05,{ }^{\# \#} P<0.01$ vs. SRS mice (two-way ANOVA with post hoc Bonferroni test). The data are presented as mean \pm SEM, $n=7-10 /$ group. Con, control; FLX, fluoxetine; SRS, space restriction stressed.
A BONT/A (0.18U)

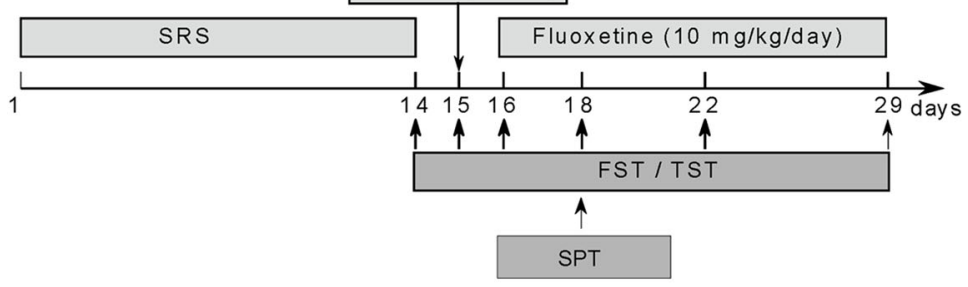

B

C

D
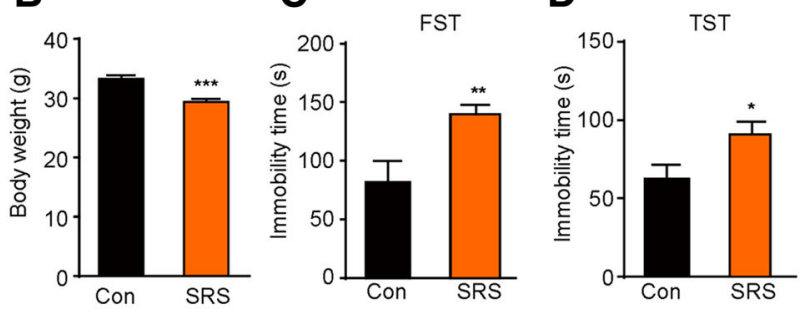

E
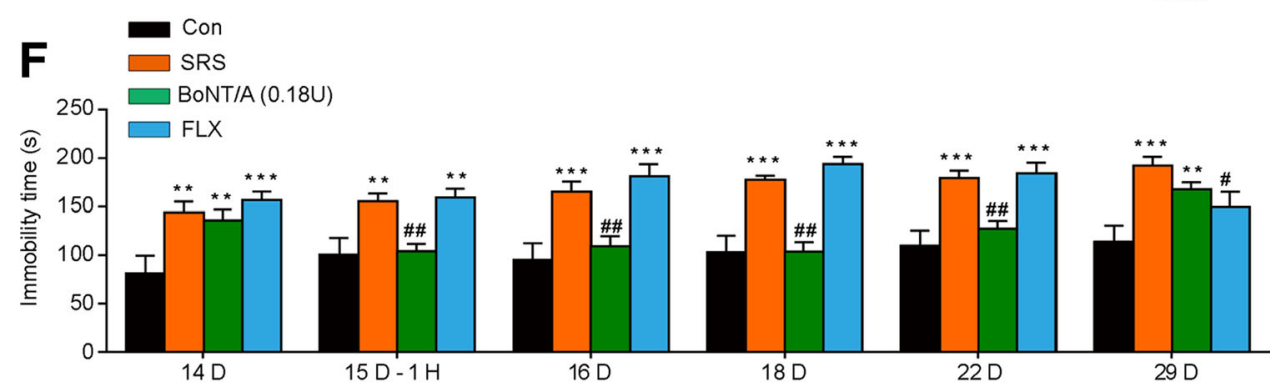

G

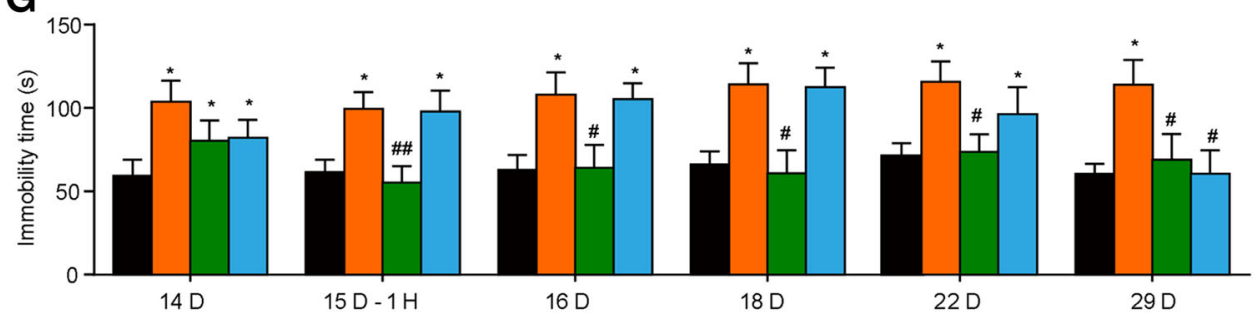

H

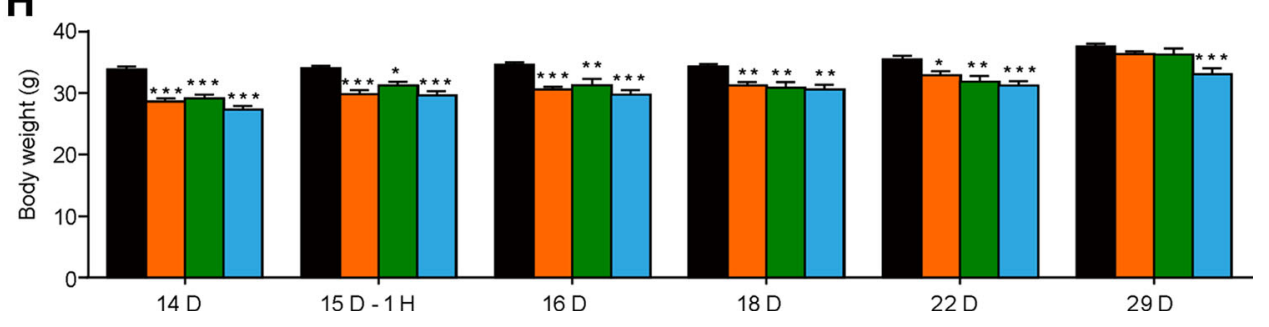

